RUfFO de FreItAS-JUNIOR ${ }^{2}$

Karine ANUSCA MARTINS ${ }^{3}$

Antônio César Pereira ${ }^{4}$

Charles Esteves Pereira ${ }^{5}$

EDÉSIO MARTINS ${ }^{6}$

Artigo Original

Palavras-chave

Palavras-chave

Linfedema

Neoplasias da mama

Ultrassonografia Doppler

Cintilografia

Keywords

Lymphedema

Breast neoplasms

Ultrasonography, Doppler

Radionuclide imaging

\section{Alterações venosas e linfáticas em mulheres com linfedema após linfadenectomia axilar no tratamento do câncer de mama}

\author{
Venous and lymphatic alterations in women with lymphedema after \\ axillary lymphadenectomy in breast cancer treatment
}

Resumo

OBJETIVO: Avaliar as alterações do sistema venoso axilo-subclávio e do sistema linfático em mulheres com linfedema após linfadenectomia axilar para o tratamento do câncer de mama. MÉTODOS: Trata-se de um estudo de série de casos, envolvendo 11 mulheres com linfedema unilateral de membro superior após linfadenectomia axilar para o tratamento do câncer de mama. $\bigcirc$ estudo foi realizado em hospital universitário do Brasil Central no período compreendido entre os meses de março de 2010 e março de 2011 . Avaliou-se a presença de alterações venosas nas veias subclávia e axilar, por meio do exame de ultrassonografia com dopplervelocimetria, e de alterações linfáticas, pela linfocintilografia, em ambos os membros superiores. O teste Exato de Fisher foi utilizado na comparação entre os membros. RESULTADOS: No membro superior com linfedema, detectou-se diferença significativa na veia subclávia, em relação ao membro contralateral, quanto ao volume do fluxo $(p<0,001)$, sendo que $54,6 \%$ das mulheres apresentaram fluxo aumentado. Já, na veia axilar, $45,4 \%$ apresentaram fluxo aumentado e 45,4\% reduzido, com diferença significante $(p<0,01)$ na comparação entre os membros. Também foram observadas alterações linfáticas significativas $(p<0,05)$, em relação ao membro contralateral, representadas pelo trajeto do vaso (não visibilizado), número de vasos linfáticos (nenhum), linfonodos axilares (ausentes) e refluxo dérmico (presente). No membro superior contralateral sem linfedema, não foram encontradas alterações venosas ou linfáticas. CONCLUSÃO: As mulheres submetidas à linfadenectomia axilar para o tratamento do câncer de mama apresentam tanto alterações venosas quanto linfáticas no membro superior com linfedema.

\section{Abstract}

PURPOSE: To evaluate changes in the venous axillary-subclavian and lymphatic systems of women with lymphedema after axillary lymphadenectomy for breast cancer treatment. METHODS: This was a case series involving 11 women with unilateral upper limb lymphedema after axillary lymphedenectomy for the treatment of breast cancer. The study was carried out in the Mastology Program of the Clinical Hospital of the Federal University of Goiás, Goiânia, GO, during the period between March 2010 and March 2011 . Doppler velocimetry ultrasonography was used to detect the presence of venous changes in the subclavian and axillary veins. Lymphatic changes were evaluated by lymphoscintigraphy in both upper limbs. Fisher's exact test was used for the comparison between limbs. RESULTS: Subclavian vein flow volume in the upper limb with lymphedema was significantly different from that in the contralateral $\operatorname{limb}(p<0.001), 54.6 \%$ of the women had increased flow. In the axillary vein, $45.4 \%$ had increased flow and $45.4 \%$ had decreased flow, with a statistically significant difference $(p<0.01)$ between limbs. Compared to the contralateral limb, significant lymphatic changes ( $p<0.05$ ) were also found in the vessel route (not visualized), number of lymphatic vessels (none), axillary lymph nodes (absent) and dermal reflux (present). In the contralateral upper limb without lymphedema, no venous or lymphatic alterations were encountered. CONCLUSION: The women subjected to axillary lymphadenectomy for the treatment of breast cancer presented both venous and lymphatic changes in the upper limb with lymphedema.
Correspondêncio

Ruffo de Freitas-Júnio Hospital das Cllinicas, Bloco B, $1^{0}$ avenida, $\mathrm{s} / \mathrm{n}$ - Setor Universitário CEP: $74606-050$ Goiânia (GO), Brasil

Recebido

$14 / 11 / 2013$

Aceito com modificacōes
Trabalho realizado na Rede Goiana de Pesquisa em Mastologia, Pós-Graduação em Ciências da Saúde, Universidade Federal de Goiás - UFG - Goiânia (GO), Brasil.

(Pontifícia Universidade Católica de Goiás - PUC/GO - Goiânia (GO), Brasil.

2Departamento de Ginecologia e Obstetrícia da Faculdade de Medicina, Universidade Federal de Goiás - UFG - Goiânia (GO), Brasil.

3Faculdade de Nutrição, Universidade Federal de Goiás - UFG - Goiânia (GO), Brasil.

${ }^{4}$ Curso de Medicina do Centro Universitário de Anápolis - UniEVANGÉlICA - Anápolis (GO), Brasil.

Instituto de Angiologia de Goiânia - IAG - Goiânia (GO), Brasil.

oRegistro de Câncer de Base Populacional de Goiânia - Goiânia (GO), Brasil.

Conflito de interesse: não há. 


\section{Introdução}

Dentre as várias morbidades que surgem após o tratamento para o câncer de mama, o linfedema é considerado uma das mais importantes, pois pode causar efeitos adversos como depressão, baixa autoestima, alteração na imagem corporal, prejuízo funcional do membro ipsilateral à cirurgia ${ }^{1}$, além de quadros de infecção ${ }^{2}$ e, ocasionalmente, linfangiossarcoma ${ }^{3}$.

A etiologia do linfedema é multifatorial e pouco compreendida, sendo a linfadenectomia axilar e a radioterapia, após tratamento para o câncer de mama, alguns dos principais fatores predisponentes, por ocasionarem lesão do sistema linfático e consequente comprometimento da sua função ${ }^{4}$. No entanto, o desenvolvimento do linfedema, secundário à neoplasia mamária, não consiste apenas na obstrução do sistema linfático, mas no somatório de diversos eventos, como idade, obesidade e infecção ${ }^{2,5,6}$.

Atualmente, um fator considerado contribuinte para a fisiopatologia do linfedema é o hemodinâmico, devido à inter-relação entre o sistema linfático e o sanguíneo, que, por sua vez, também pode sofrer alterações estruturais e funcionais após o tratamento ${ }^{7,8}$.

Diante do exposto, este estudo teve como objetivo avaliar as alterações do sistema venoso axilo-subclávio e do sistema linfático em mulheres com linfedema após linfadenectomia axilar para o tratamento do câncer de mama.

\section{Métodos}

Trata-se de um estudo de série de casos, envolvendo 11 mulheres com linfedema unilateral de membro superior após linfadenectomia axilar para o tratamento do câncer de mama.

As participantes foram selecionadas no período compreendido entre os meses de março de 2010 e março de 2011, no Programa de Mastologia (PM), do Hospital das Clínicas (HC), da Universidade Federal de Goiás (UFG), em Goiânia, Goiás. Foram encaminhadas ao serviço de fisioterapia, nesse período, 14 mulheres com diagnóstico de linfedema, após tratamento para o câncer de mama. Dessas, 11 se enquadraram nos critérios de inclusão e aceitaram fazer parte da pesquisa, confirmando sua participação por meio da assinatura do Termo de Consentimento Livre e Esclarecido (TCLE).

Os critérios de inclusão foram: mulheres com idade superior a 18 anos; que foram submetidas ao tratamento cirúrgico para o câncer de mama, seja ele a mastectomia radical modificada (Patey ou Madden) ou a ressecção segmentar, associado à linfadenectomia axilar; que haviam recebido tratamento cirúrgico há mais de seis meses da data da entrevista e que apresentavam linfedema unilateral de membro superior. Como critérios de exclusão, mulheres operadas bilateralmente; que apresentavam infecção no braço ou com incapacidade de responder as perguntas.

O estudo foi avaliado pelo Comitê de Ética em Pesquisa Médica Humana e Animal, do Hospital das Clínicas da Universidade Federal de Goiás, e aprovado sob o protocolo n ${ }^{\circ}$ 161/2009, em 03 de dezembro de 2009.

Os dados da pesquisa foram colhidos por uma única avaliadora capacitada na área de fisioterapia oncológica. As características sociodemográficas foram obtidas por meio de entrevista, por ocasião da entrada da participante no estudo. A idade foi considerada em anos e estratificada em menor ou igual a 40 anos e maior que 40 anos; a escolaridade foi caracterizada em analfabetas, ensino fundamental, médio, superior e pós-graduação; e estratificou-se a profissão em do lar e fora do lar .

As características clínicas também foram obtidas na entrada do estudo, por meio de exame físico. O peso foi colhido no momento da avaliação e utilizou-se balança eletrônica; a altura foi medida com uma fita métrica com extensão de $2 \mathrm{~m}$ e precisão de $0,1 \mathrm{~cm}$, afixada a uma parede sem rodapé; calculou-se o índice de massa corporal a partir da fórmula peso/altura'; e a pressão arterial foi aferida duas vezes, sendo a medida final a média dos referidos valores, com a mulher sentada e o braço apoiado sobre a mesa. Considerou-se hipertensão arterial os valores iguais ou superiores a $140 / 90 \mathrm{mmHg}$ ou se a mulher fizesse uso de medicação anti-hipertensiva9 .

O tempo e tipo de cirurgia, outros tratamentos relacionados ao câncer de mama e a avaliação do linfedema foram obtidos por meio de exame físico e consulta prévia aos prontuários das participantes.

O critério utilizado para o diagnóstico de linfedema foi a perimetria, realizada bilateralmente, com uma única fita métrica. Foram realizadas três marcações no braço e antebraço, em posição supina, de cinco em cinco centímetros, acima e abaixo da prega cubital, respectivamente. As marcações foram ajustadas de acordo com o tamanho do membro de cada participante e colheu-se a circunferência acima do ponto demarcado. Caracterizou-se linfedema quando pelo menos uma das medidas do membro homolateral à cirurgia foi superior a $2 \mathrm{~cm}$ em relação ao membro contralateral ${ }^{10}$.

O exame de linfocintilografia foi realizado com injeção subdérmica de $1 \mathrm{mCi}(37 \mathrm{MBq})$ de Dextrantecnécio ${ }^{99 \mathrm{~m}}$, no $3^{\circ}$ espaço interdigital de ambos os membros superiores. O mapeamento linfocintilográfico foi realizado uma e três horas após a injeção subdérmica, aplicada por um único técnico qualificado. As imagens foram registradas em câmara de cintilação de alta resolução, modelo Option, MCD, AC, forte AC. Fabricante Philips-ADAC, Laboratories, 540 Alder Drive, Milpitas, CA-USA, 95035, com processamento pelo programa 
Pegasys versão 5.0. Aos 60 e 180 minutos, foram obtidas as imagens em varredura, com os membros superiores ao longo do corpo, e as imagens estáticas, aos 60 minutos, com os membros superiores em semiflexão de cotovelo ${ }^{11}$.

A análise qualitativa das imagens linfocintilográficas foi realizada por um único médico especializado, que não havia tido contato prévio com as participantes. Os parâmetros analisados bilateralmente foram: trajeto do vaso (linear, tortuoso ou não visibilizado); número de vasos linfáticos (único, múltiplos ou nenhum); presença de linfonodos axilares e epitrocleares; presença de refluxo dérmico e circulação colateral ${ }^{12}$.

O exame de ultrassonografia com dopplervelocimetria foi realizado por um único médico cirurgião vascular, com experiência nessa área de atuação. Durante o exame, a mulher foi posicionada em decúbito dorsal, com o membro superior em rotação externa e abdução a 90 graus. As veias subclávia e axilar, de ambos os membros superiores, foram avaliadas com varredura dinâmica, por meio de aparelho de ultrassonografia da marca Philips, modelo HD11 XE e transdutor linear com frequência de 5-12 MHz. Posicionou-se o transdutor nos eixos transversal e longitudinal do vaso com ângulo de 60 graus. $\mathrm{Na}$ varredura transversal, avaliaram-se o calibre, a morfologia e a compressibilidade do vaso; já no eixo longitudinal, avaliaram-se o funcionamento valvular e as curvas de fluxo com dopplervelocimetria pulsada ${ }^{13}$.

As variáveis analisadas, bilateralmente, pelo exame de dopplervelocimetria foram ${ }^{8,13}$ : ausência da veia, definida pela não identificação do vaso durante o exame; trombo, caracterizado pela coagulação do sangue no interior do vaso sanguíneo; perda do fluxo, ausência do fluxo no segmento avaliado; estenose, presença de estreitamento do calibre vascular; compressibilidade venosa, definida pela diminuição do volume do vaso mediante uma pressão ou força; perda da fasicidade do fluxo com a respiração, caracterizada pela perda do efeito modulador dos movimentos respiratórios sobre o fluxo venoso; imobilidade da parede venosa durante a respiração (quando não se observa movimento da parede do vaso durante a respiração); alteração do volume do fluxo, tendo como parâmetro de referência o membro contralateral sem linfedema e, para o cálculo do volume, utilizou-se a fórmula $\mathrm{V}=\mathrm{AxVMx} 60$ (onde: $\mathrm{V}$ é o volume, expresso em cm; A é a área, expressa em $\mathrm{cm}^{2}$; VM é a velocidade média, expressa em $\mathrm{cm} / \mathrm{s}$ e presença de circulação colateral, definida pelo aumento da rede de vasos próximo ao local de obstrução de um vaso maior.

Utilizou-se estatística descritiva para análise dos dados por meio do pacote estatístico Statistical Package for Social Sciences (SPSS), versão 18.0, nas quais as variáveis qualitativas foram expressas em frequências e percentuais e as variáveis quantitativas, em média e desvio padrão (DP). Foram calculadas as taxas percentuais das alterações evidenciadas pela ultrassonografia com dopplervelocimetria e pela linfocintilografia. Utilizou-se o teste Exato de Fisher na comparação entre os membros, considerando o nível de significância de $\mathrm{p}<0,05$.

\section{Resultados}

A média de idade das 11 mulheres participantes do estudo foi de 48,6 $( \pm 6,25)$ anos e variou de 37 a 56 anos. Em relação às características sociodemográficas e clínicas, observou-se que 8/11 apresentaram escolaridade de nível médio ou mais e que a maioria exercia atividades fora do lar, era obesa e não tinha hipertensão arterial (Tabela 1).

Segundo relato das participantes, o tempo de aparecimento do edema de membro superior (suposto linfedema), após o tratamento cirúrgico para o câncer de mama até a data de avaliação pela fisioterapeuta responsável, variou de 4 a 111 meses (9,2 anos), com uma média de 45,9 $( \pm 37,1)$ meses.

No que diz respeito às técnicas cirúrgicas, para o tratamento do câncer de mama, verificou-se que a cirurgia de ressecção segmentar foi mais frequente, com predominância do lado direito. Todas as mulheres foram submetidas ao esvaziamento axilar completo (níveis I-III), à radioterapia adjuvante, em tecido mamário remanescente (plastrão) da mastectomia, porém nenhuma participante recebeu radiação na região axilar. Todas as pacientes também receberam quimioterapia adjuvante (Tabela 1 ).

Em relação ao tratamento fisioterapêutico, observou-se que nenhuma participante havia realizado Terapia Física Complexa (TFC) ou tratamento com luvas de contenção elástica (de forma isolada). A maioria dos casos foi tratada com drenagem linfática manual (DLM) e apenas uma referiu a associação da DLM e o uso de luva de contenção elástica. Quase $30 \%(\mathrm{n}=3)$ não receberam qualquer tipo de tratamento fisioterapêutico até o momento da avaliação (Tabela 1). É importante ressaltar, ainda, que as participantes do estudo não se submeteram aos tratamentos fisioterapêuticos prescritos de forma contínua. Os parâmetros linfocintilográficos do membro superior sem linfedema apresentaram-se normais em todas as mulheres. Na região do braço, observaram-se trajeto do vaso linear, número de vasos linfáticos únicos, presença dos linfonodos axilares e ausência de refluxo dérmico e circulação colateral. No antebraço, o trajeto do vaso foi linear, número de vasos linfáticos únicos, os linfonodos epitrocleares não estavam presentes em 8/11 das mulheres, variação essa considerada normal, e não se observou presença de refluxo dérmico e circulação colateral (Tabela 2). No membro superior com linfedema, observaram-se parâmetros de anormalidades do sistema linfático. 
$\mathrm{Na}$ região do braço, a maioria apresentou trajeto do vaso não visibilizado e nenhum número de vasos linfáticos. Os linfonodos axilares também não foram visualizados em nenhum caso e a presença de refluxo dérmico foi evidenciada em seis delas. Tais parâmetros apresentaram diferença significativa quando comparados com o braço contralateral sem linfedema. A ausência da circulação colateral foi o único parâmetro que se manteve dentro da normalidade, sem diferença significativa (Tabela 2).

No antebraço, em 6/11 das mulheres avaliadas, o trajeto do vaso não foi visibilizado. Quanto ao número de vasos linfáticos, nenhum foi identificado; e o refluxo dérmico estava presente na maioria dos casos. Esses achados apresentaram diferença significativa quando comparados com o antebraço contralateral. Quanto aos linfonodos epitrocleares, observou-se que, em 10/11 mulheres, estavam ausentes, variação considerada normal. A circulação colateral apresentou-se ausente em todos os casos. Tais parâmetros não foram significativos quando comparados com o membro sem linfedema (Tabela 2).

No que se refere à análise das alterações venosas das veias subclávia e axilar, pela dopplervelocimetria, não foi encontrada nenhuma anormalidade anatômica nos membros superiores sem linfedema. Em contrapartida, nos membros superiores com linfedema, observou-se a presença dessas alterações, tanto na veia subclávia como na axilar.

As principais alterações da veia subclávia foram: imobilidade da parede venosa durante a respiração e alteração no volume do fluxo, sendo que 6/11 das mulheres apresentaram fluxo aumentado e cinco delas, fluxo reduzido. Já na veia axilar, verificou-se estreitamento do calibre vascular, imobilidade da parede venosa durante a respiração
Tabela 1. Distribuição das características sociodemográficas, clínicas e relacionadas aos tratamentos

\begin{tabular}{|c|c|c|}
\hline Variáveis & Categorias & n (11) \\
\hline \multirow[t]{2}{*}{ Idade } & $\leq 40$ anos & 1 \\
\hline & $>40$ anos & 10 \\
\hline \multirow[t]{5}{*}{ Escolaridade } & Analfabeto & 1 \\
\hline & Ensino fundamental & 2 \\
\hline & Ensino médio & 3 \\
\hline & Superior & 4 \\
\hline & Pós-graduaç̃̃o & 1 \\
\hline \multirow[t]{2}{*}{ Profissão } & Do lar & 3 \\
\hline & Fora do lar & 8 \\
\hline \multirow[t]{3}{*}{ IMC } & Eutrofia & 4 \\
\hline & Sobrepeso & 1 \\
\hline & Obesidade & 6 \\
\hline HAS & $\begin{array}{l}\text { Sim } \\
\text { Não }\end{array}$ & $\begin{array}{l}4 \\
7\end{array}$ \\
\hline $\begin{array}{l}\text { Lado da cirurgia / Membro } \\
\text { com linfedema }\end{array}$ & $\begin{array}{l}\text { Direito } \\
\text { Esquerdo }\end{array}$ & $\begin{array}{l}8 \\
3\end{array}$ \\
\hline Linfadenectomia axilar & Nível I-III & 11 \\
\hline Tipos de cirurgias & $\begin{array}{l}\text { Mastectomia com preservação do } \\
\text { músculo peitoral maior } \\
\text { Mastectomia com preservação } \\
\text { dos dois músculos peitorais } \\
\text { Ressecção segmentar }\end{array}$ & $\begin{array}{l}1 \\
3\end{array}$ \\
\hline Radioterapia adjuvante & $\begin{array}{c}\text { Mama remanescente / Plastrão } \\
\text { da mastectomia } \\
\text { Região axilar }\end{array}$ & $\begin{array}{l}11 \\
00\end{array}$ \\
\hline Quimioterapia & Sim & 11 \\
\hline $\begin{array}{l}\text { Tratamentos } \\
\text { fisioterapêuticos }\end{array}$ & $\begin{array}{c}\text { DLM } \\
\text { DLM e luva de contenção elástica } \\
\text { Nenhum }\end{array}$ & $\begin{array}{l}7 \\
1 \\
3\end{array}$ \\
\hline
\end{tabular}

IMC: índice de massa corporal; HAS: hipertensão arterial sistêmica; DLM: drenagem linfática manual.

Tabela 2. Distribuição dos achados linfocintilográficos dos membros superiores com e sem linfedema (Goiânia-G0, 2012)

\begin{tabular}{|c|c|c|c|c|c|c|c|c|c|c|}
\hline \multirow[b]{2}{*}{ Variáveis } & \multicolumn{4}{|c|}{ Braço } & \multirow[b]{2}{*}{ Valor $p$} & \multicolumn{4}{|c|}{ Antebraço } & \multirow[b]{2}{*}{ Valor $\mathrm{p}$} \\
\hline & \multicolumn{2}{|c|}{ Com linfedema } & \multicolumn{2}{|c|}{ Sem linfedema } & & \multicolumn{2}{|c|}{ Com linfedema } & \multicolumn{2}{|c|}{ Sem linfedema } & \\
\hline \multicolumn{11}{|l|}{ Trajefo do vaso } \\
\hline Tortuoso & 00 & 0,0 & 02 & 18,2 & \multirow[t]{2}{*}{$0,004^{*}$} & 00 & 0,0 & 01 & 9,1 & \multirow[t]{2}{*}{$0,013^{*}$} \\
\hline Não visibilizado & 07 & 63,6 & 00 & 0,0 & & 06 & 54,6 & 00 & 0,0 & \\
\hline \multicolumn{11}{|c|}{ Número de linfáticos } \\
\hline Nenhum & 07 & 63,6 & 00 & 0,0 & $0,004^{*}$ & 06 & 54,6 & 00 & 0,0 & $0,013^{*}$ \\
\hline \multicolumn{11}{|c|}{ Linfonodos epitrocleares } \\
\hline $\begin{array}{l}\text { Presente } \\
\text { Ausente }\end{array}$ & - & - & - & - & - & $\begin{array}{l}01 \\
10\end{array}$ & $\begin{array}{r}9,1 \\
90,9\end{array}$ & $\begin{array}{l}03 \\
08\end{array}$ & $\begin{array}{l}27,3 \\
72,7\end{array}$ & $0,293^{*}$ \\
\hline \multicolumn{11}{|l|}{ Linfonodos axilares } \\
\hline $\begin{array}{l}\text { Presente } \\
\text { Ausente }\end{array}$ & $\begin{array}{l}00 \\
11\end{array}$ & $\begin{array}{r}0,00 \\
100,0\end{array}$ & $\begin{array}{l}11 \\
00\end{array}$ & $\begin{array}{r}100,0 \\
0,0\end{array}$ & $<0,001^{*}$ & - & - & - & - & - \\
\hline \multicolumn{11}{|l|}{ Circulação colateral } \\
\hline $\begin{array}{l}\text { Presente } \\
\text { Ausente } \\
\end{array}$ & $\begin{array}{l}00 \\
11 \\
\end{array}$ & $\begin{array}{r}0,0 \\
100,0 \\
\end{array}$ & $\begin{array}{l}00 \\
11 \\
\end{array}$ & $\begin{array}{r}0,0 \\
100,0 \\
\end{array}$ & $1,00^{*}$ & $\begin{array}{l}00 \\
11 \\
\end{array}$ & $\begin{array}{r}0,0 \\
100,0 \\
\end{array}$ & $\begin{array}{l}00 \\
11 \\
\end{array}$ & $\begin{array}{r}0,00 \\
100,0 \\
\end{array}$ & $1,00^{*}$ \\
\hline
\end{tabular}

Exame de linfocintilografia; *Teste exato de Fisher 
e volume do fluxo alterado, sendo que 5/11 apresentaram fluxo aumentado e 5/11, reduzido. Ao comparar o membro com e sem linfedema, observou-se diferença significativa, tanto para a veia subclávia quanto para a axilar, somente em relação ao volume do fluxo (Tabela 3).

\section{Discussão}

No que se refere à técnica cirúrgica para o tratamento do câncer de mama, alguns autores consideram que quanto mais agressiva, maiores são os riscos de desenvolver linfedema ${ }^{14,15}$, o que não foi confirmado por outros. No entanto, alguns trabalhos não encontraram diferença significativa entre a cirurgia empregada e o aparecimento do linfedema ${ }^{16,17}$. Com a dissecção axilar extensa, a incidência do linfedema aumenta, por favorecer a obstrução e sobrecarga funcional do sistema linfático ${ }^{5,18}$.

A radioterapia, na mama residual ou no plastrão da mastectomia, não é considerada um fator de risco para o linfedema ${ }^{5}$ e, sim, a radioterapia axilar, que leva ao bloqueio e à compressão dos vasos linfáticos, devido à fibrose ${ }^{19}$.

O tratamento sistêmico com quimioterapia não é considerado por alguns autores como um fator da gênese do linfedema ${ }^{5,20}$. No entanto, outros estudos demonstraram haver tal associação $0^{19,21,22}$.

Dentre as formas de tratamento do linfedema, a TFC é o tratamento fisioterapêutico mais recomendado, por possibilitar melhor resposta do quadro ${ }^{23}$. Contudo, para o alcance de bons resultados terapêuticos, é fundamental a adesão das pacientes às medidas preconizadas ${ }^{24}$.

Para avaliar o sistema linfático, incluindo a confirmação clínica do diagnóstico de linfedema periférico, a linfocintilografia é um exame de grande confiabilidade, de fácil realização e pouco invasivo ${ }^{12}$, que permite a visualização dos vasos linfáticos e linfonodos, além de quantificar o transporte linfático ${ }^{25}$.
Os parâmetros linfocintilográficos qualitativos que caracterizam a presença de linfedema envolvem desde a assimetria ou não visibilidade dos vasos linfáticos a assimetria ou ausência dos linfonodos regionais, presença de refluxo dérmico e canais linfáticos colaterais ${ }^{12,26}$.

As mulheres que desenvolvem linfedema, após cirurgia para o tratamento do câncer de mama, podem apresentar as alterações linfáticas supracitadas em decorrência da dissecção $\operatorname{axilar}^{26}$ e da radioterapia ${ }^{27}$. Esses achados assemelham-se aos encontrados no presente estudo, exceto pelo fato de que não foram observados casos com presença de circulação colateral.

Alguns autores afirmam que alterações funcionais e anatômicas pré-existentes do sistema linfático podem aumentar a predisposição ao linfedema após dissecção dos linfonodos axilares ${ }^{27,28}$. Isso, possivelmente, se deve ao fato de essas alterações interferirem nos mecanismos compensatórios utilizados por esse sistema para restabelecer o fluxo da linfa, que, por sua vez, encontra-se alterado devido ao tratamento ${ }^{26}$.

Como parâmetro de ausência de alterações linfáticas preexistentes (padrão de normalidade) avaliou-se, no presente trabalho, o membro superior contralateral após a cirurgia, apesar de a realização prévia da linfocintilografia não ter sido objeto de estudo. No entanto, nenhum dos casos avaliados apresentou alterações do sistema linfático no membro contralateral, sugerindo-se, pois, que o desenvolvimento do linfedema ipsilateral à cirurgia pode ou não ter sofrido influência de disfunções linfáticas anteriores.

Os sistemas sanguíneo e linfático possuem íntima relação e dependem um do outro para a manutenção do equilíbrio entre os processos de filtração e absorção, por isso a presença de disfunções em um deles poderá alterar a função do outro ${ }^{23,29}$. Por essa razão, alguns pesquisadores discutem que as alterações hemodinâmicas também predispõem ao linfedema ${ }^{7,8,15}$. Pois, apesar da dissecção dos linfonodos axilares ${ }^{17}$ e da radioterapia serem consideradas

Tabela 3. Distribuição das alterações de membro superior das veias subclávia e axilar das mulheres participantes do estudo

\begin{tabular}{|c|c|c|c|c|c|c|c|c|c|c|}
\hline \multirow{3}{*}{ Variáveis } & \multicolumn{4}{|c|}{ Veia subclávia (membro superior) } & \multirow{3}{*}{ Valor $\mathrm{p}$} & \multicolumn{4}{|c|}{ Veia axilar (membro superior) } & \multirow{3}{*}{ Valor $p$} \\
\hline & \multicolumn{2}{|c|}{ Com linfedema } & \multicolumn{2}{|c|}{ Sem linfedema } & & \multicolumn{2}{|c|}{ Com linfedema } & \multicolumn{2}{|c|}{ Sem linfedema } & \\
\hline & n & $\%$ & n & $\%$ & & n & $\%$ & $\mathrm{~N}$ & $\%$ & \\
\hline \multicolumn{11}{|c|}{$\begin{array}{l}\text { Imobilidade da parede } \\
\text { venosa durante a respiração }\end{array}$} \\
\hline Sim & 01 & 9,1 & 00 & 0,0 & $0,50^{\star}$ & 01 & 9,1 & 00 & 0,0 & $0,50^{\star}$ \\
\hline \multicolumn{11}{|c|}{$\begin{array}{l}\text { Estreitamento do calibre } \\
\text { vascular (estenose) }\end{array}$} \\
\hline Sim & 00 & 00,0 & 00 & 0,0 & - & 01 & 9,1 & 00 & 0,0 & $0,50^{\star}$ \\
\hline \multicolumn{11}{|l|}{ Volume do fluxo } \\
\hline $\begin{array}{l}\text { Normal } \\
\text { Reduzido } \\
\text { Aumentado }\end{array}$ & $\begin{array}{l}00 \\
05 \\
06\end{array}$ & $\begin{array}{r}0,0 \\
45,4 \\
54,6\end{array}$ & $\begin{array}{l}11 \\
00 \\
00\end{array}$ & $\begin{array}{r}100,0 \\
0,0 \\
0,0\end{array}$ & $<0,001^{*}$ & $\begin{array}{l}01 \\
05 \\
05\end{array}$ & $\begin{array}{r}9,1 \\
45,4 \\
45,5\end{array}$ & $\begin{array}{l}11 \\
00 \\
00\end{array}$ & $\begin{array}{r}100,0 \\
0,0 \\
0,0\end{array}$ & $<0,001^{*}$ \\
\hline
\end{tabular}

Exame de ultrassonografia com dopplervelocimetria; ${ }^{*}$ Teste exato de Fisher. 
as principais causas da gênese do linfedema ${ }^{14}$, tais procedimentos por si só não explicam a razão de algumas mulheres desenvolverem ou não o edema linfático ${ }^{30}$.

As alterações linfáticas e o linfedema são temas de diversas pesquisas; no entanto, são poucos os estudos que contemplam a associação entre as alterações vasculares e o linfedema ${ }^{7,8,15}$. Até o momento, não há um consenso a respeito da influência hemodinâmica no linfedema, principalmente em relação ao fator venoso.

Em estudo com ultrassonografia com dopplervelocimetria, os autores observaram, em 81 mulheres com linfedema após tratamento para o câncer de mama, $57 \%$ de obstrução venosa e $14 \%$ de congestão venosa, e concluíram que essas alterações estavam relacionadas diretamente à presença do linfedema ${ }^{8}$.

Outro estudo, que avaliou a presença de alteração venosa em mulheres com ( $n=39)$ e sem $(n=16)$ linfedema, após linfadenectomia axilar, não detectou nenhuma anormalidade ${ }^{31}$. Em pesquisa posterior, estudiosos observaram a presença de estenose na veia axilar em mulheres com e sem linfedema, submetidas à dissecção dos linfonodos axilares ${ }^{15}$.

No presente estudo, foram encontradas anormalidades anatômicas nas veias subclávia e axilar, como imobilidade da parede venosa durante a respiração e estreitamento do calibre vascular, no membro superior com linfedema, porém sem significância estatística se comparadas com o membro contralateral.

Outra anormalidade encontrada foi em relação ao volume do fluxo venoso, em que a maioria das mulheres apresentou fluxo aumentado na veia subclávia e quase a metade, na veia axilar, ao se comparar com o membro contralateral. Esses achados são consistentes com outro estudo, em que os autores inferiram aumento do fluxo venoso no membro com linfedema ${ }^{8}$. Recentemente, em uma pesquisa que utilizou ultrassonografia com dopplervelocimetria portátil, os pesquisadores também encontraram aumento no fluxo da veia axilar; contudo, em pacientes sem linfedema submetidas à dissecção axilar ${ }^{7}$.
Além disso, observou-se, no presente trabalho, que 5/11 das mulheres apresentaram redução do fluxo venoso nas veias subclávia e axilar no membro superior com linfedema. Esses dados corroboram com outros achados, em que os autores encontraram diminuição do retorno venoso e do volume do fluxo em pacientes com linfedema $(n=11)$, quando comparados a indivíduos saudáveis $(\mathrm{n}=13)^{32}$. Alguns pesquisadores acreditam que essa alteração ocorre em razão da ruptura mecânica ou fibrose do vaso como resultado da cirurgia ${ }^{17}$.

Como possíveis limitações desse estudo, pode-se mencionar: conclusões que se fundamentam em poucos casos, o que dificultou a validação da associação causal; e a necessidade de um Grupo Controle para viabilizar outras análises de associação.

\section{Conclusão}

As alterações do sistema venoso axilo-subclávio do membro superior com linfedema foram: volume do fluxo aumentado (veia subclávia) e equivalência entre o volume do fluxo aumentado e reduzido (veia axilar). Já as alterações do sistema linfático foram: trajeto do vaso (não visibilizado); número de linfáticos (nenhum); linfonodos axilares e refluxo dérmico (ausentes).

A realização do presente estudo permitiu um maior conhecimento das alterações venosas e linfáticas em mulheres com linfedema de membro superior após linfadenectomia axilar. No entanto, sugere-se a realização de estudos de coorte ou caso controle, preferencialmente multicêntricos, que poderão estabelecer, de forma mais evidente, a relação dessas variáveis com o desenvolvimento do linfedema.

\section{Agradecimentos}

Sarah Patrizia Araújo Valinote recebeu bolsa CAPES (Coordenação de Aperfeiçoamento Pessoal de Nível Superior) e Ruffo Freitas-Júnior recebeu apoio do Instituto Avon.

\section{Referências}

1. Alegrance FC, Souza CB, Mazzei RL. Qualidade de vida e estratégias de enfrentamento em mulheres com e sem linfedema pós-câncer de mama. Rev Bras Cancerol. 2010;56(3):341-51.

2. Freitas-Júnior R, Esperidião $M D$, Holanda $M E$, Esperidião $A C$, Godoy CS. Severe erysipelas after treatment for breast cancer. Breast J. 2005; 11 (1):76-7.

3. Ocaña A, Delgado C, Rodríguez CA, Bellido L, Izquierdo N, Martín $R$, et al. Case 3. Upper limb lymphangiosarcoma following breast cancer therapy. J Clin Oncol. 2006;24(9):1477-8.
4. Rezende LF, Rocha AVR, Gomes CS. Avaliação dos fatores de risco no linfedema pós-tratamento de câncer de mama. J Vas Bras. 2010;9(4):233-8.

5. Meeske KA, Sullivan-Halley J, Smith AW, McTiernan A, Baumgartner $K B$, Harlan LC, et al. Risk factors for arm lymphedema following breast cancer diagnosis in black women and white women. Breast Cancer Res Treat. 2009; 1 13(2):383-91.

6. Tsai R, Dennis LK, Lynch CF, Snetselaar LG, Zamba GK, ScottConner $C$. The risk of developing arm lymphedema among breast 
cancer survivors: a meta-analysis of treatment factors. Ann Surg Oncol. 2009; 16(7): 1959-72.

7. Nascimben Matheus C, Guirro ECO. Change in blood flow velocity demonstrated by Doppler ultrasound in upper limb after axillary dissection surgery for the treatment of breast cancer. Breast Cancer Res Treat. $2011 ; 127(3): 697-704$.

8. Svensson WE, Mortimer PS, Tohno E, Cosgrove DO. Colour Doppler demonstrates venous flow abnormalities in breast cancer patients with chronic arm swelling. Eur J Cancer. 1994;30A(5):657-60.

9. Bergmann A. Prevalência de linfedema subsequente a tratamento cirúrgico para câncer de mama [Internet]. Rio de Janeiro: Escola Nacional de Saúde Pública; 2000 [citado 2010 Dez 5]. Disponível em: <http://portalteses.icict.fiocruz.br/pdf/FIOCRUZ/2000/ bergmannam/pdf/capa.pdf>

10. Freitas Júnior R, Ribeiro LFJ, Taia L, Kajita D, Fernandes MV, Queiroz GS. Lymphedema in breast cancer patients submitted to modified radical mastectomy. Rev Bras Ginecol Obstet. 2001 ;23(4):205-8. Portuguese.

11. Yuan Z, Chen L, Luo Q, Zhu J, Lu H, Zhu R. The role of radionuclide lymphoscintigraphy in extremity lymphedema. Ann Nucl Med. 2006;20(5):34 1-4.

12. Szuba A, Shin WS, Strauss HW, Rockson S. The third circulation: radionuclide lymphoscintigraphy in the evaluation of lymphedema. J Nucl Med. 2003;44(1):43-57.

13. Maffei FHA, Lastória S, Yoshida WB, Rollo HA, Giannini M, Moura R. Doenças vasculares periféricas. 4. ed. Rio de Janeiro: Guanabara Koogan; 2008.

14. Hayes SC, Janda M, Cornish B, Battistutta D, Newman B. Lymphedema after breast cancer: incidence, risk factors, and effect on upper body function. J Clin Oncol. 2008;26(21):3536-42.

15. Pain SJ, Vowler S, Purushotham AD. Axillary vein abnormalities contribute to development of lymphoedema after surgery for breast cancer. Br J Surg. 2005;92(3):311-5.

16. Freitas-Silva R, Conde DM, Freitas-Júnior R, Martinez EZ. Comparison of quality of life, satisfaction with surgery and shoulder-arm morbidity in breast cancer survivors submitted to breast-conserving therapy or mastectomy followed by immediate breast reconstruction. Clinics (São Paulo). 2010;65(8):781-7.

17. Britton TM, Buczacki SJ, Turner CL, Vowler SL, Pain SJ, Purushotham $A D$. Venous changes and lymphoedema 4 years after axillary surgery for breast cancer. Br J Surg. 2007;94(7):833-4.

18. McLaughlin SA, Wright M, Morris KT, Giron GL, Sampson MR, Brockway JP, et al. Prevalence of lymphedema in women with breast cancer 5 years after sentinel lymph node biopsy and axillary dissection: objective measurements. J Clin Oncol. 2008;26(32):5213-9.

19. Paiva DM, Leite ICG, Rodrigues VO, Cesca MG. [Associated factors of lymphedema in breast cancer patients]. Rev Bras Ginecol Obstet. $2011 ; 33(2): 75-80$. Portuguese.
20. Bani HA, Fasching PA, Lux MM, Rauh C, Willner M, Eder I, et al. Lymphedema in breast cancer survivors: assessment and information provision in a specialized breast unit. Patient Educ Couns. 2007;66(3): $311-8$

21. Bevilacqua JL, Kattan MW, Changhong Y, Koifman S, Mattos $I E$, Koifman R, et al. Nomograms for predicting the risk of arm lymphedema after axillary dissection in breast cancer. Ann Surg Oncol. 2012;19(8):2580-9.

22. Shah C, Wilkinson JB, Baschnagel A, Ghilezan M, Riutta J, Dekhne $\mathrm{N}$, et al. Factors associated with the development of breast cancer related lymphedema after whole-breast irradiation. Int J Radiat Oncol Biol Phys. 2012;83(4): 1095-100.

23. Leduc $A$, Leduc $O$. Drenagem linfática: teoria e prática. 3a ed. São Paulo: Manole; 2007.

24. Panobianco MS, Parra MV, Almeida AM, Prado MAS, Magalhães PAP. Estudo da adesão às estratégias de prevenção e controle do linfedema em mastectomizadas. Esc Anna Nery Rev Enferm. 2009;13(1):161-8.

25. Rezende LF, Pedras FV, Ramos CD, Gurgel MSC. Avaliação das compensações linfáticas no pós-operatório de câncer de mama com dissecção axilar através da linfocintilografia. J Vasc Bras. 2008;7(4):370-5.

26. Suami H, Pan WR, Taylor Gl. Changes in the lymph structure of the upper limb after axillary dissection: radiographic and anatomical study in a human cadaver. Plast Reconstr Surg. 2007; 120(4):982-91.

27. Rezende LF, Pedras FV, Ramos CD, Gurgel MS. Evaluation of lymphatic compensation by lymphoscintigraphy in the postoperative period of breast cancer surgery with axillary dissection. Tumori. $2011 ; 97(3): 309-15$.

28. Rezende LF, Pedras FV, Ramos CD, Gurgel MSC. Função linfática do membro superior no pré-operatório de câncer de mama. Rev Assoc Med Bras 2011;57(5):540-4.

29. Bernas $M$, Witte $M$, Kriederman B, Summers P, Witte C. Massage therapy in the treatment of lymphedema. Rationale, results, and applications. IEEE Eng Med Biol Mag. 2005;24(2):58-68.

30. Stanton AW, Modi S, Mellor RH, Levick JR, Mortimer PS. Recent advances in breast cancer-related lymphedema of the arm: lymphatic pump failure and predisposing factors. Lymphat Res Biol. $2009 ; 7(1): 29-45$.

31. Yildirim E, Soydinç P, Yildirim N, Berberoglu U, Yüksel E. Role of increased arterial inflow in arm edema after modified radical mastectomy. J Exp Clin Cancer Res. 2000; 19(4):427-30.

32. Montgomery LD, Dietrich MS, Armer JM, Stewart BR, Ridner $\mathrm{SH}$. Segmental blood flow and hemodynamic state of lymphedematous and nonlymphedematous arms. Lymphat Res Biol. $2011 ; 9(1): 31-42$. 